\title{
COLABORADORES
}

\section{Carlos J. Aldazábal}

Doctor en Ciencias Sociales y Magíster en Comunicación y Cultura por la Universidad de Buenos Aires. Su tesis sobre cultura popular y música folclórica argentina, El aire estaba quieto, se publicó en 2009, a través de un premio del Fondo Nacional de las Artes. En poesía publicó, entre otros títulos El banco está cerrado (2010), Hain, el mundo selknam en poesía historieta (2012, con ilustraciones de Eleonora Kortsarz), Piedra al pecho (2013) y Las visitas de siempre (2014). Es docente del Seminario de Cultura Popular y Masiva en la Carrera de Ciencias de la Comunicación de la Universidad de Buenos Aires.

carlosaldarabal@gmail.com

\section{Lidia Isabel Almeida Reyes}

Licenciada en Psicología Educativa; Magíster en Educación Superior; docente de la Facultad de Filosofía, Letras y Ciencias de la Educación; Carrera de Educación Parvularia, Universidad Central del Ecuador.

lidiaisabelalmeidareyes@gmail.com

\section{Fausto E. Ávalos Cascante}

Profesor principal 1 EPN, jefe del departamento de Física EPN, director de la Escuela de Ciencias EPN, decano de la Facultad de Ciencias EPN, director de investigación EPN, director de la REDU, director de investigación de la REDU director del laboratorio de UAVs y APLICACIONES ÓPTICAS EPN, coordinador del Proyecto FAE-EPN, coordinador del proyecto IEE-EPN, profesor invitado PUC-RIO BR USP Sao Carlos, Publicaciones, artículos, ponencias, evaluados de proyectos e innovaciones tecnológicas SENASCYT. Entre otras actividades.

eduardo.avalos@epn.edu.ec

\section{Oswaldo Báez Tobar}

Biólogo graduado en la Universidad Central del Ecuador. Fue profesor principal de Zoología, Biología Evolutiva, Biología del Desarrollo y Anatomía Comparada de Vertebrados, facultades de Filosofía y Ciencias Médicas de la Universidad Central. Ex profesor de la Universidad Católica de Quito. Trabajó en conservación de la naturaleza y el medio ambiente en la Fundación Natura, Ministerio del Ambiente y el Consejo de Manejo Forestal, FSC (por sus siglas en inglés). Ha sido presidente de la Sociedad Ecuatoriana de Biología en varios períodos. Coautor de varios libros, autor de varios artículos científicos y numerosos artículos de divulgación en medios impresos y virtuales nacionales e internacionales.

oswaldobaez@hotmail.com 


\section{Dimitri Barreto Vaquero}

Médico - Psiquiatra; ex Decano de la Facultad de Ciencias Médicas; profesor jubilado de Psiquiatría en la Facultad de Ciencias Médicas; actualmente es Director de la Cátedra de Cultura Universal en la Universidad Central del Ecuador.

dimbarretov@yahoo.com

\section{Fabián Bedón Samaniego}

UNESCO-Ecuador. Sociólogo mención en Relaciones Internacionales por la Pontificia Universidad Católica del Ecuador. Maestría en Gestión de Proyectos por la Escuela Superior Politécnica del Litoral. Actualmente es Coordinador de Proyectos Sector Cultura en UNESCO-Ecuador.

f.bedon@unesco.org

\section{Juan Cadena}

Matemático, Director del Proyecto Etnomatemática, Facultad de Filosofía, Letras y Ciencias de la Educación.

jcadena3581@gmail.com

\section{Alí Calderón}

Doctor en Letras Mexicanas por la Benemérita Universidad Autónoma de Puebla (México); Licenciado en lingüística y literatura hispana. Poeta, narrador y ensayista. Ha colaborado con reseñas y poesías en Alforja, Dosfilos, Luvina, Reverso, Revista de Literatura Mexicana Contemporánea (University of Texas, en El Paso) y Tierra Adentro. Profesor de la Facultad de Filosofía y Letras en la Benemérita Universidad Autónoma de Puebla. Es cofundador de la editorial y la gacetilla Círculo de Poesía. Becario de la Fundación para las Letras Mexicanas, 2003-2004. Premio Interuniversitario (BUAP/UDLA/UIA) de Oratoria del estado de Puebla, 2003. Premio Nacional de Poesía Ramón López Velarde, 2004. Premio Latinoamericano de Poesía Benemérito de las Américas, 2007, por Ser en el mundo. Parte de su obra se incluye en diversas compilaciones románticas y de poesías eróticas, entre ellas Los mejores poemas mexicanos, edición 2005 (2005); Más vale sollozar afilando la navaja (2004) y Poesía ante la incertidumbre, antología de nuevos poetas en español (2011).

alicalderonf@hotmail.com

\section{Edison Franklin Cando Vaca}

Licenciado en Artes; Tecnología en Recreación Infantil; Magíster en Gerencia de Proyectos Educativos Sociales. Trabaja en la Universidad Central del Ecuador, Facultad de Filosofía, Letras y Ciencias de la Educación, Carrera de Educación Parvularia. 


\section{José Luis Comparán Elizondo}

Licenciado en Ciencias Físico Matemáticas, egresado de la Facultad de Ciencias Físico Matemáticas de la Universidad Autónoma de Nuevo León en el año de 1968. Obtuvo el grado de Maestro en Ciencias, con Especialidad en Geofísica, en el Departamento de Física de la Universidad de Toronto, en Toronto, Ontario, Canadá. En el año de 1973 obtuvo el grado científico de Doctor en Ciencias Pedagógicas, en la Universidad de Camagüey, en Camagüey, República de Cuba. En el año 2005. Se desempeñó como Director de la Facultad de ciencias Físico Matemáticas de la Universidad de Nuevo león de Octubre de 1974 a Julio de 1978, y en ese período, específicamente en el año de 1975 crea la carrera de Lic. en Ciencias Computacionales en la Facultad de Ciencias Físico Matemáticas de la Universidad Autónoma de Nuevo León.

$$
\text { jlcomparane@gmail.com }
$$

\section{Hólger Paúl Córdova Vinueza}

Profesor de la Facultad de Jurisprudencia, Ciencias Políticas y Sociales, Universidad Central del Ecuador. Autor de libros y artículos en temas de innovación democrática, derechos de participación, democracia, cambio constitucional y poder popular. Ha colaborado profesionalmente en entidades públicas y privadas sobre la gestión de experiencias y procesos participativos. Ha participado como docente en algunas universidades del país, fue Director del Centro de Estudios Construyendo Ciudadanía y Democracia de la Universidad Central del Ecuador y es investigador del Centro Andino de Estudios Estratégicos.

hpaulcordova@gmail.com

\section{Mónica Alexandra Coronel Silva}

Ingeniera Civil de la Universidad Central del Ecuador. Investigadora del Centro de Excelencia en Transporte Intermodal y Fluvial, CETIF - Universidad Central del Ecuador

mcoronel@uce.edu.ec

\section{Andrea Cote-Botero}

Es autora de los libros de poemas: Desierto Rumor (2016), La ruina que nombro (2015), Puerto Calcinado, Cosas Frágiles y Chinatown a toda hora (Libro Objeto). Ha publicado además los libros en prosa: Una fotógrafa al desnudo: biografía de Tina Modottiy Blanca Varela o la escritura de la soledad. Culminó su doctorado en literatura hispanoamericana en la Universidad de Pennsylvania. Ha obtenido los reconocimientos: Premio Nacional de Poesía de la Universidad Externado de Colombia en el año 2003, Premio Internacional de Poesía Puentes de Struga (2005) y el Premio Cittá de Castrovillari Prize (2010) a Porto in Cenere, version italiana de Puerto Calcinado. Es profesora de la maestría bilingue en escritura creativa en la Universidad de Texas en El Paso. 


\section{Sergio Espinoza Proa}

Licenciado en Antropología Social por la ENAH (México, 1977), Especialista en Investigación Educativa por la UAEM (Cuernavaca, 1987), Maestro en Filosofía e Historia de las Ideas por la UAZ (Zacatecas, 1992) y Doctor en Filosofía por la Universidad Complutense de Madrid (Madrid, 1997). Investigador y docente de la Universidad Autónoma de Zacatecas. Ganador del decimotercer Concurso Internacional de Ensayo, convocado conjuntamente por Siglo XXI Editores, la Universidad Autónoma de Sinaloa y el Colegio de Sinaloa, con la obra Del saber de las musas. La filosofía y el fenómeno arte.

sproa52@hotmail.com

\section{Carmen Fernández Galán}

Doctora en Humanidades y Artes por la Universidad Autónoma de Zacatecas. Líneas de investigación: edición crítica de textos del siglo XVIII, historia literaria, hermenéutica y semiótica. Publicaciones: Syzigias y quadraturas lunares... (Factoría-UAZ, 2010) y Obelisco para el ocaso de un príncipe (Texere-UAZ, 2011).

carmenfgalan@gmail.com

\section{José Ignacio Gallego Revilla}

$\mathrm{PhD}$ en Arqueología y Antropología por la Universidad Complutense de Madrid (realización de tesis). Diploma de Estudios Avanzados, Prehistoria y Arqueología por la Universidad Complutense de Madrid). Licenciatura en Historia, Prehistoria y Arqueología por la Universidad Complutense de Madrid. Arqueólogo profesional. Consultor UNESCO. Museo de Segovia.

nachogare@hotmail.com

\section{Facundo Gómez}

Profesor de la Universidad de Buenos Aires, Argentina. Sus ensayos sobre literatura han sido publicados en importantes medios especializados.

vfagomez_27@yahoo.com.ar

\section{Francisco González de la Fuente}

Licenciado en Historia por la Universidad Complutense de Madrid, Magíster en Historia Medieval de Castilla y León por la UBU, USAL, UVA y ULE.

fjg117@gmail.com

\section{Salomón Jaya}

Director del Centro de Excelencia en Transporte Intermodal y Fluvial CETIF- Profesor Principal de la Facultad de Ingeniería, Ciencias Físicas y Matemática de la UCE. 


\section{Mario S. López Flores}

Ingeniero en Electrónica y Control por la Escuela Politécnica Nacional, en el año 2015. Entre su formación complementaria se encuentra el idioma inglés, seminarios de Calidad de la Energía y Óptica en la EPN.

\section{Luis A. Manzano Orbe}

Realizó sus estudios de tercer nivel de Ingeniería Electrónica y Control en la Escuela Politécnica Nacional entre 2009 y 2014. Sus intereses se centran en Inteligencia Artificial y Redes Neuronales, Electrónica aplicada a la Acústica y Óptica.

\section{Cecilia del Carmen Marcillo}

Doctora en Psicología Industrial, Magíster en Neurosicología Infantil; docente de pre y posgrado de la Facultad de Ciencias Psicológicas; tutora de tesis de pre y Posgrado de la Facultad de Ciencias Psicológicas; coordinadora del Proyecto de "Fortalecimiento del neurodesarrollo en los niños de 0 a 3 años de los CIBV de las parroquias rurales de la mancomunidad de Pedro Moncayo"; ex Directora de la Carrera de Psicología Infantil y Psicorrehabilitación; profesional de la Neuropsicología en la Consulta Privada.

cemarcillo@yahoo.com

\section{Iván Oñate}

Director de ANALES, Universidad Central del Ecuador; ex Profesor de la Facultad de Filosofía, Letras y Ciencias de la Educación, Universidad Central del Ecuador

Licenciatura en Sociología y Ciencias Politicas, Universidad Central del Ecuador; Doctorado en Ciencias de la Información (Semiótica), Universidad Autónoma de Barcelona (España); profesor invitado por: Westminster University, Kings College of London y University of London, Texas A\&M University, Florida State University, Universidad de Lieja (Bélgica), Universidad de Lille 3 (Francia), Universidad de Lovaina (Bélgica), Universidad de Austin Texas, Universidad Autónoma de México, Universidad Autónoma de Guanajuato, Universidad Autónoma de Nuevo León (México), Universidad Autónoma de San Luis Potosí (México), Universidad Nacional de Colombia, Universidad Libre de Colombia. Su obra ha sido traducida al alemán, francés, inglés, portugués, griego, polaco e italiano.

$$
\text { ivonate@mac.com }
$$

\section{Edwin Robinson Panchi Culqui}

Licenciado en Idiomas Inglés y Francés; Licenciado en Música; Magíster en Docencia Universitaria y Administración; Magister en Pedagogía e Investigación Musical. Trabaja en la Universidad Central del Ecuador - Facultad de Filosofía, Letras y Ciencias de la Educación, Carrera de Educación Parvularia.

erobinpan69@yaboo.com 


\section{Paúl Puma}

Poeta, dramaturgo, guionista, editor, crítico literario y docente de la Carrera de Lenguaje y Literatura de la Facultad de Filosofía, Letras y Ciencias de la Educación, Universidad Central del Ecuador.

paul3puma@yahoo.com

\section{Abraham Quezada Vergara}

$\mathrm{PhD}$ en Estudios Americanos. Profesor de Historia y Geografía. Escritor y docente universitario, actualmente es Primer Secretario y se desempeña en la Embajada de Chile en Ecuador. Paralelo a su actividad profesional, es investigador en temas de historia contemporánea de Chile. Ha publicado varios libros y artículos tanto en medios nacionales como extranjeros. Entre ellos, sobresale su trabajo Pedro Aguirre Cerda o la trayectoria de un ideal educativo, obra ganadora del primer lugar a nivel nacional organizado por la Universidad de Chile en 1990 y un Diccionario de conceptos históricos y geográficos de Chile en el 2004 (con reediciones). Especialista en la vida y obra de Pablo Neruda destacándose la publicación de artículos y libros como Pablo Neruda, Epistolario viajero, 1927-1973, editado en 2004.

aquezadav@minrel.gov.cl

\section{Nelson Gustavo Rodríguez Aguirre}

Vicerrector Académico y de Investigación. Maestría en Desarrollo de Talento Humano; Doctor en Psicología Clínica; Investigador en el Área de Ciencia, Tecnología y Sociedad. Autor de varios libros y artículos.

viceacad@uce.edu.ec

\section{Alejandro Rodríguez Rodrigo}

Arqueólogo profesional, Municipalidad de Colta. Especialización Superior en Gestión de la Cultura, con mención en Patrimonio, Universidad Andina Simón Bolívar, Ecuador (2012). Diploma de Enseñanza del Español como Lengua Extranjera, Universidad Complutense de Madrid, España (2009). Acceso a la condición de Militar de Complemento adscrito al Cuerpo de Especialistas de la Armada Española, Escuela Naval Militar (2004-2005). Curso de Actitud Pedagógica, Universidad de Valencia, España (2002). Licenciado en Historia y Arqueología, Universidad de Valencia, España (1995-2001).

aroro@alumni.uv.es

\section{Pablo Romo M.}

Docente de la Carrera de Ciencias del Lenguaje y Literatura de la Facultad de Filosofía, Letras y Ciencias de la Educación, Universidad Central del Ecuador.

pabloromo007@yahoo.com 


\section{Rolando Sáenz}

Matemático de la Universidad Nacional de Colombia y Doctor en Matemáticas de la Universidad de Montpellier, Francia. Ha sido profesor de matemáticas de la Escuela Politécnica Nacional y de la Universidad Central del Ecuador, institución en la que ha ocupado numerosos cargos directivos. Entre sus publicaciones e investigaciones se destacan numerosos libros universitarios de matemáticas y la dirección de varios proyectos relacionados con modelos matemáticos para la industria hidrocarburífera.

rsaenz@uce.edu.ec; rolando.saenz@epn.edu.ec

\section{Saadia Sánchez Vegas}

Socióloga por la Universidad de Zulia (Venezuela) con títulos de Maestría en Library Science por la San Jose State University (EE.UU) y Doctorado (Ph.D.) en Library and Information Studies por la University of California, Berkeley (EE. UU.). Actualmente ocupa el cargo de Directora de la Oficina de UNESCO en Quito y Representante para Bolivia, Colombia, Ecuador y Venezuela.

quito@unesco.org

\section{Alcira Sandoval Ruiz}

Oficina de UNESCO en Quito y Representante para Bolivia, Colombia, Ecuador y Venezuela. Especialista de Programas y Responsable del Sector Cultura Quito/Cultura.

a.sandoval-ruiz@unesco.org

\section{Raúl Serrano Sánchez}

Escritor, periodista y ensayista ecuatoriano. Ha publicado varios, cuentos y novelas, por los que ha ganado varios reconocimientos tales como el premio Nacional Joaquín Gallegos Lara en 1997. Ejerce la docencia en la Universidad Simón Bolívar en Ecuador. Algunos de sus de sus libros más conocidos son En la ciudad se ha perdido un novelista: La narrativa de Vanguardia de Humberto Salvadory Rondando a Jota Jota, inspirada en la vida del cantautor Julio Jaramillo.

raul.serrano@uasb.edu.ec

\section{Guillermo Terán Acosta}

Universidad Central del Ecuador. Coordinador de la Unidad de Física gteran30@hotmail.com

\section{Jordi Tresserras Juan}

Universitat de Barcelona/IBERTUR - Red de Patrimonio, Turismo y Desarrollo Sostenible; Consultor UNESCO-Quito Oficina Representación para Bolivia, Colombia, Ecuador y Venezuela. 
juan@ub.edu

\section{Jorge Trujillo}

Antropólogo, Coordinador de Investigación del Proyecto Etnomatemática, Universidad Central del Ecuador.

\section{Leonardo Wild}

Representante mundial de la Educación No Directiva. Entre sus libros más importantes están: Oro en la selva (novela, 1996); Unemotion (novela), escogida por Deutschlandradio y la revista Focus como una de las mejores novelas de marzo de 1997 (Unemotion fue publicada como Yo Artificial, o el futuro de las emociones, en octubre 2012); Die Insel die es nie gab (novela, 1997, junto con Frederik Hetmann); Orquídea negra o el factor vida (novela, 1999); EXTREM! (novela, 2001), El caso de los muertos de risa (novela, 2001), Cotopaxi, alerta roja (novela, 2006). Libros de ensayos: Ecología al rojo vivo (1997), recibió Mención de Honor del Premio «Isabel Tobar Guarderas»; El dinero o la vida, una guía práctica para la alquimia monetaria (Ecuador, Mayor Books, 2011); Indianermärchen aus Südamerika-Antología de cuentos indígenas de América del Sur, junto con Frederik Hetmann (Fischertaschenbuch Verlag, Alemania, 1996). Director y guionista de los documentales CEPAS, Pesta, y Yasuní, dos segundos de vida, este último ganador del Premio Mérito a la Conciencia, Los Angeles 2011.

dlwild@gmail.com

\section{Israel Zambrano B.}

UNESCO-Ecuador. Consultor de Bienes Muebles para el "Plan de Gestión para el Conjunto Conventual de San Francisco de Quito”. Licenciado en Restauración y Museología.

asra84@hotmail.com 


\section{Instrucciones para la publicación de artículos en la Revista Anales}

ANALES de la Universidad Central del Ecuador, primera revista de divulgación científica del país desde 1883, invita a la comunidad universitaria (investigadores, docentes y estudiantes, nacionales o extranjeros) a presentar artículos de investigación, ensayos y reseñas para su edición Nro. 374. Todos los escritos deberán seguir las normas que más adelante se detallan y remitirse a la siguiente dirección electrónica: revista.anales@uce.edu.ec

ANALES es una publicación dirigida a difundir las ciencias, las tecnologías y las artes. Es un medio de promoción para sus investigadores y para invitados de las universidades nacionales e internacionales. Todos sus criterios editoriales se conforman de acuerdo con los requisitos académicos y editoriales de la Secretaria de Educación Superior, Ciencia, Tecnología e Innovación (SENESCYT).

\section{Instrucción para autores}

Son requisitos indispensables para los colaboradores:

\section{De contenido:}

1. Todos los artículos, ensayos y reseñas deben ser originales y no haber sido publicados con anterioridad, así como no deben estar sometidos al mismo tiempo a dictamen en cualquier otro impreso. Además el autor cede los derechos exclusivos de publicación a la revista ANALES.

2. Los artículos o ensayos científicos deberán ser del área de las ciencias humanas y sociales, a saber: artículos referentes a análisis o polémicas sobre teorías contemporáneas, hechos sociales o debates actuales que enriquezcan y ofrezcan una nueva perspectiva teórica a las diversas disciplinas de las ciencias sociales; trabajos de divulgación científica resultado de investigaciones, que podrán ser estudios de caso, reflexión científica o ensayo científico; estudios de caso actuales o con una perspectiva histórica (regionales, nacionales o internacionales) que sean de interés general; análisis de teorías clásicas que permitan enriquecer las actuales. En el caso de las disciplinas estético-literarias deberán ser de actualidad y originalidad propias.

3. Es imprescindible entregar un resumen de una extensión de entre 100 y 150 palabras, además de anexar palabras clave del texto, todo en el idioma castellano e inglés.

4. Todos los trabajos serán sometidos a dictamen de pares ciegos a cargo del Consejo Editorial de ANALES de la Universidad Central del Ecuador, el cual está compuesto por prestigiados académicos de instituciones nacionales e internacionales. Cada trabajo será enviado a dos dictaminadores según el área de especialización disciplinaria que corresponda.

5. Los resultados de los dictámenes son inapelables. 
6. Los procesos de dictamen están determinados por el número de artículos en lista de espera. El editor de la revista informará a cada uno de los autores del avance de su trabajo en el proceso de dictamen y edición en su caso.

7. Cada número de la revista se integrará con los trabajos que en el momento del cierre de edición cuenten con la aprobación de, por lo menos, dos árbitros o dictaminadores. No obstante, con el fin de dar una mejor composición temática a cada número, ANALES se reserva el derecho de adelantar o posponer los artículos aceptados.

8. La dirección editorial de la revista se reserva el derecho de hacer la corrección de estilo y cambios editoriales que considere necesarios para mejorar el trabajo.

9. Todo caso no previsto será resuelto por el Consejo Editorial.

\section{De formato:}

1. Se aceptarán trabajos con una extensión de hasta 11000 caracteres, máximo 20 páginas incluyendo gráficos, tablas, notas a pie de página y bibliografía, en tamaño de papel A4 (21x29.7), márgenes derecho, izquierdo, superior e inferior de $2.0 \mathrm{~cm}$. Todo el texto debe estar escrito a doble espacio y alineado a la izquierda. El tamaño de letra debe ser 12 Times, estilo de fuente normal. Las reseñas deben tener una extensión de 3 a 5 páginas.

2. Todas las colaboraciones deberán entregarse en archivo electrónico, en procesador Word, sin ningún tipo de formato, sangrías o notas automáticas.

3. En la portada del trabajo deberá aparecer el nombre completo del/los autor/es.

4. Los cuadros, tablas y gráficos deben presentarse agrupados al final del documento y en los programas informáticos correspondientes para diseño gráfico. En el texto se debe señalar el lugar dónde habrán de colocarse; asimismo, deben ser elaborados y enviados en archivos aparte en algún programa de hoja de cálculo, preferiblemente en Excel. Imágenes o fotografías deben enviarse con respaldo aparte (formato jpg de alta resolución).

5. Las notas a pie de página deberán ser únicamente aclaratorias o explicativas, es decir, han de servir para ampliar o ilustrar lo dicho en el cuerpo del texto, y no para indicar las fuentes bibliográficas, ya que para eso está la bibliografía. Cabe señalar que esta deberá contener las referencias completas de las obras de los autores que se citen en el cuerpo del texto, sin agregar otras que no sean citadas.

6. Las citas deberán usar el sistema APA.

7. La bibliografía debe estar escrita en el mismo sistema, ordenada alfabética y cronológicamente según corresponda. No usar mayúsculas continuas. Los apellidos y nombres de los autores deben estar completos, es decir, no deben anotarse sólo abreviaturas. 


\section{Título}

El título debe ser claro, conciso y escogido cuidadosamente para reflejar el contenido del trabajo reportado. No utilizar abreviaturas.

\section{Nombre(s) del autor(es) y afiliación(es)}

Para listar autores del trabajo, poner el primer nombre y el/los apellido(s) de todos los investigadores que han hecho una sustancial contribución al trabajo. El nombre del autor principal irá en primer lugar y al final el nombre del tutor, o director del proyecto. Deben omitirse todos los títulos, ocupaciones y grados académicos como: Prof., Coord., Lcdo., MSc, PhD.

Después de los autores poner la afiliación (nombre y dirección de la institución) de cada uno y con letras superíndices relacionar la afiliación con los autores. Señalar el contacto o a quien va dirigida la correspondencia con un asterisco $\left(^{*}\right)$ sobre el apellido y agregar el correo electrónico. Si el autor a quien va dirigida la correspondencia ya no está en la institución donde el trabajo fue realizado, colocar la dirección actual como una nota al pie de página marcada con un asterisco $(*)$.

\section{Resumen}

Todos los manuscritos deben tener un resumen del trabajo realizado. Contiene una clara indicación del objetivo, los resultados más importantes y las conclusiones para que los lectores puedan determinar si el texto completo será de su interés. Debe estructurarse en un solo párrafo, no debe exceder las 200 palabras.

\section{Palabras claves}

Colocar de tres a cinco palabras claves

\section{Title}

Poner el título del manuscrito en inglés. A continuación colocar la palabra "Abstract." Y escribir el contenido del resumen en inglés. Después, escribir "Key words." Y poner las palabras claves en inglés.

Envío de trabajos:

ANALES Universidad Central del Ecuador

Quito - Ecuador

Telf.: (+593 7) 2526493-Ext. 12

Correo electrónico: revista.anales@uce.edu.ec 